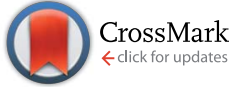

Cite this: RSC Adv., 2017, 7, 8670

Received 2nd December 2016 Accepted 21st January 2017

DOI: $10.1039 / c 6 r a 27657 a$

rsc.li/rsc-advances

\title{
In situ determination of mechanical properties for poly(ether ether ketone) film under extreme conditions
}

\begin{abstract}
Jiayu Wang, ${ }^{a}$ Qiang Zhou, ${ }^{a}$ Danming Chao, ${ }^{b}$ Fangfei Li ${ }^{\star a}$ and Tian Cui ${ }^{a}$
The mechanical properties of special engineering plastic have been intensively studied in recent years. However, there are few studies on the mechanical variation under extreme conditions. In this study, we chose poly(ether ether ketone) (PEEK) to study its mechanical behavior under high temperature and high pressure using Brillouin scattering coupled with an electrical resistance heating technique and diamond anvil cell device. The isothermal compressibility and pressure dependence of the mechanical moduli of PEEK film were examined and determined. A typical negative thermal expansion phenomenon was observed under high temperature and high pressure, which has been explained and discussed by virtue of free volume theory. Comprehensive investigation of elastic properties as a function of pressure not only provides an effective way to understand the mechanical behavior of PEEK under high temperature and high pressure, but also supplies an available theoretical direction for their practical application under extreme conditions
\end{abstract}

\section{Introduction}

The versatility of polymers has allowed them to be used extensively under extreme conditions, as various components in explosives, aerospace, submarine and heavy machinery, where the polymers witness high strain rates, high pressure, and even high temperature. ${ }^{1-11}$ Therefore, the deep knowledge of their mechanical properties under extreme conditions is essential for design and development of polymer components. Up to now, several approaches have been explored to achieve this goal. Takamatsu and coworkers obtained polyimide Hugoniot data up to $0.6 \mathrm{TPa}$ with good accuracy. The laser-driven equation-ofstate (EOS) experiments were performed with emission measurements from the rear sides of a shocked target at a high laser intensity with $2.5 \mathrm{~ns}$ duration. ${ }^{1}$ The dynamical behavior of polyimide was also investigated in detail using a flyer driven by chemical explosion. The plots of pressure vs. volume behind shock waves were measured in the pressure range of 0-56 GPa. ${ }^{2}$ Moreover, various theoretical models have also been applied for the evaluation of extensive EOS data of pure polymers, polymersolvent and polymer blends. ${ }^{12-17}$

Recently, some polymer samples, such as polyolefins, polysiloxanes, and polyimide, have been investigated by high pressure Brillouin scattering spectroscopy technique, ${ }^{3-11}$ which is a nondestructive method to provide insight into the elastic and

${ }^{a}$ State Key Lab of Superhard Materials, College of Physics, Jilin University, Changchun 130012, P. R. China. E-mail: lifangfei@jlu.edu.cn

${ }^{b}$ Alan G. MacDiarmid Institute, College of Chemistry, Jilin University, Changchun, 130012, P. R. China bulk mechanical properties of optically transparent materials by measuring their acoustic velocities. The diamond anvil cell (DAC) supplies the high pressure condition. The elastic properties and mechanical moduli of these polymers under high pressure were determined accurately, which provided an effective theoretical direction for their utility under extreme condition. Contrast to the previous approaches, high pressure Brillouin scattering spectroscopy method was considered as the optimal approach, due to its accuracy, effectiveness and safety.

Poly(ether ether ketone) (PEEK), as one of the most used special engineering plastic, has attracted special attention due to its high stability, excellent mechanical strength, good radiation-resistant. ${ }^{18-20}$ These characteristics make PEEK an excellent candidate material for specific applications under extreme conditions. To the best of our current knowledge, there is no research on the determination of mechanical properties of PEEK under high pressure, much less the simultaneous high temperature. Herein, the isothermal compressibility and pressure dependence of mechanical moduli were determined for the aromatic PEEK through high temperature high pressure Brillouin scattering upto $520 \mathrm{~K}$ and $13.9 \mathrm{GPa}$.

\section{Experimental}

PEEK APTIV 2000 series film with thickness of $50 \mu \mathrm{m}$ (VICTREX, $T_{\mathrm{g}}: 143{ }^{\circ} \mathrm{C}, \rho: 1.26 \mathrm{~g} \mathrm{~cm}^{-3}$, the structure of PEEK was shown in the Fig. 1) is used as the research object enclosed in a symmetric DAC with a large conical opening aperture. The culet size of diamond anvils is $400 \mu \mathrm{m}$ in diameter. Silicon oil was utilized as the pressure transmission medium in DAC to minimize the 


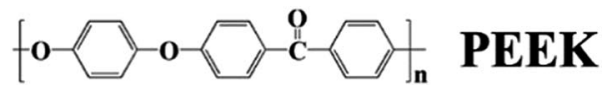

Fig. 1 The molecular structure of poly(ether ether ketone).

pressure gradient. ${ }^{21-23} \mathrm{~A}$ small annealed ruby chip was loaded into the chamber for the pressure calibration. Brillouin scattering experiments were performed in symmetric platelet scattering geometry. The DAC places to bisect this scattering angle such that the difference vector, i.e., the acoustic phonon wave vector, is in the plane of the sample. A single-frequency $532 \mathrm{~nm}$ laser was used as Brillouin excitation source. The Brillouin spectra were collected by $3+3$ pass tandem Fabry Perot interferometer designed by Sandercock. ${ }^{24,25}$ Ruby fluorescence was recorded through Raman spectroscopy system using Acton SpectraPro 500i spectrometer with a liquid nitrogen-cooled CCD detector (Princeton Instruments). An electrical resistance heater was set around the gasket clipped between diamond anvils, a thermal couple was fixed on one side of diamond about $500 \mu \mathrm{m}$ away from the sample chamber for temperature calibration. The temperature was controlled by a feedback power holding a fluctuation within $1 \mathrm{~K}$.

\section{Results and discussion}

In this study, Brillouin scattering technology was applied on the PEEK films in symmetric platelet geometry. The Brillouin shift $(\Delta \nu)$ was converted to sound velocity $(v)$ using the relative equation:

$$
v=\frac{\lambda_{0} \Delta \nu}{2 \sin \left(\frac{\theta}{2}\right)}
$$

here, $\lambda_{0}$ is the incident laser wavelength, and $\theta$ is the scattering angle. In this experiment, $\theta$ is fix at $60^{\circ}$, which is benefit for velocity measurements from the frequency shift under in situ high pressure high temperature conditions due to the unnecessary of refractive index data. ${ }^{24}$

Brillouin spectra of PEEK film under high pressures at $300 \mathrm{~K}$ were shown in Fig. 2. Both longitudinal $\left(V_{\mathrm{L}}\right)$ and transverse $\left(V_{\mathrm{T}}\right)$ sound velocities were clearly observed at high pressures, but their intensity decreased remarkably with increasing pressure. Finally, the shear modes disappear at $13.9 \mathrm{GPa}$. In addition, a pair of back scattering signals $\left(V_{180^{\circ}}\right)$ can be detected below 0.4 GPa. However, they would gradually outflow the spectral range of $20 \mathrm{GHz}$ with increasing of the applied pressure. To confirm the isotropy of the PEEK film, the direction dependence of acoustic velocities for PEEK film has been checked under 0, 0.4 and $6.1 \mathrm{GPa}$ at $300 \mathrm{~K}$, then the resulting velocity variations are presented in Fig. 3a. Obviously, the enclosed PEEK film exhibits highly isotropic at various conditions. Although some little fluctuations with angle rotation were observed at elevated pressure, they all fall within the range of the maximum error of the peak position. Furthermore, X-ray diffraction (XRD) measurement on PEEK film was also performed under ambient pressure at $300 \mathrm{~K}$. A typical broad peak was obtained and

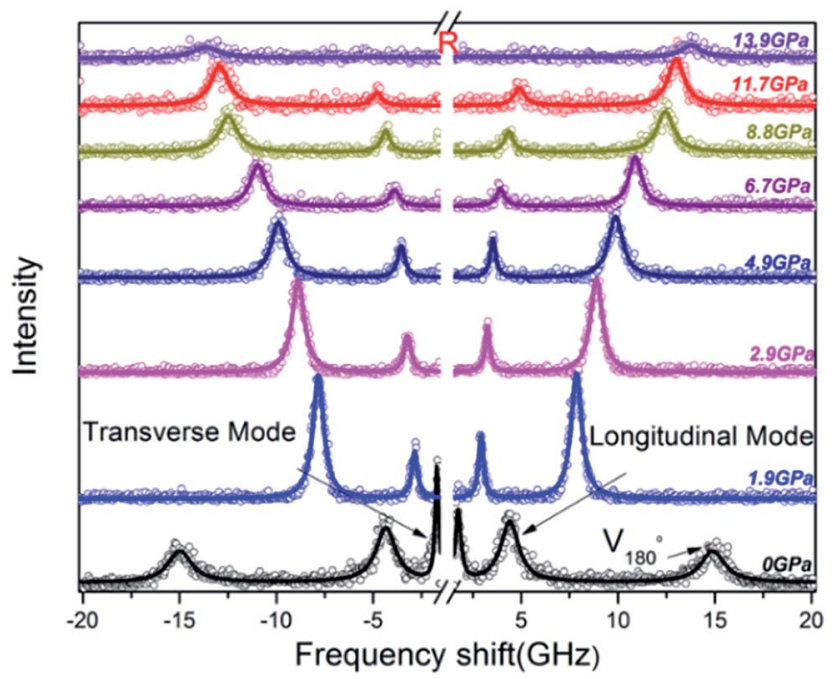

Fig. 2 Selected Brillouin spectra of PEEK film in platelet scattering geometry at $300 \mathrm{~K}$. The center $R$ is due to inelastic Rayleigh scattering. $V_{180^{\circ}}$ is the backscattering signal.

presented in Fig. 3b, which is the characteristic of amorphous material. Therefore, in our measurement of Brillouin spectra for PEEK film, the velocity is collected at only one direction at elevated temperatures and pressures.

Next, we measured the temperature dependence of velocity of PEEK film under ambient pressure, as shown in Fig. 4. The soften of sound velocity for the PEEK film is observed, the longitudinal velocity decreases about $30 \%$ from $300 \mathrm{~K}$ to $500 \mathrm{~K}$, meanwhile the transverse velocity decreases $15 \%$. While a little pressure less than $0.1 \mathrm{GPa}$ is applied on the sample, the velocity soften phenomenon derived from high temperature is suppressed (Fig. 4), which indicated that the soften phenomenon would be ignored after the application of pressure. Due to the increase of full width at half maximum with temperature changing, we draw out the scope of the maximum error of the peak position to describe its trend of changes with temperature.

The pressure dependence of velocity along several isothermals, including $300,380,430,480$ and $520 \mathrm{~K}$, are presented in Fig. 5. We observed that acoustic velocities of sample increase smoothly with increasing pressure at a constant temperature. However, they decrease slightly with increasing temperature at a constant pressure. Furthermore, the shear velocity is lost near $13.9 \mathrm{GPa}$ at $300 \mathrm{~K}$, while they are lost at relative lower pressure at high temperatures, such as losing around $9.5 \mathrm{GPa}$ at $520 \mathrm{~K}$.

Based on the measured velocities, the bulk sound velocity $V_{\mathrm{b}}$ was calculated according to following equation:

$$
V_{\mathrm{b}}^{2}=V_{\mathrm{L}}^{2}-\frac{4}{3} V_{\mathrm{T}}^{2}
$$

As shown in the Fig. 5, the resultant bulk velocity increases remarkably with the elevated pressure. But slight decreases of the bulk velocity was observed with increasing temperature. Then the densities were derived from the bulk sound velocity through numerical integration of eqn (3) 

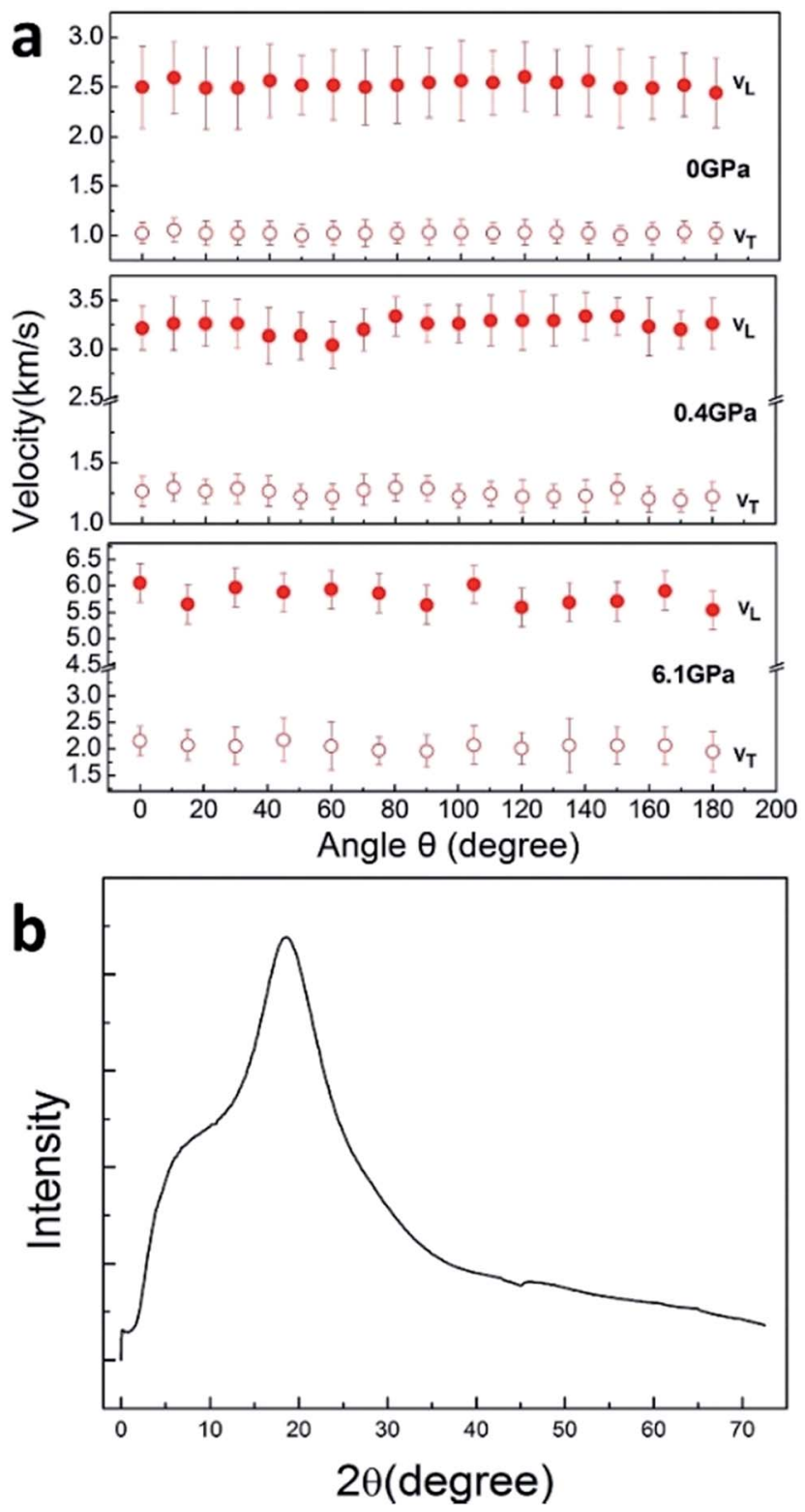

Fig. 3 (a) Direction dependence of acoustic velocities for PEEK film under $0,0.4$ and $6.1 \mathrm{GPa}$ at $300 \mathrm{~K}$, longitudinal and transverse velocities are denoted by $V_{\mathrm{L}}$ and $V_{\mathrm{T}}$, respectively. (b) XRD pattern of PEEK film under ambient condition.

$$
\rho-\rho_{0}=\int_{\rho_{0}}^{\rho} \frac{\gamma}{V_{\mathrm{b}}^{2}} \mathrm{~d} p
$$

where $\rho$ represents the density at a given pressure, $\rho_{0}$ is the density at ambient pressure, and $\gamma$ is the ratio of isobaric and isochoric specific heats. As $\gamma$ is known to vary between 1 and 1.2 for polymers, the ratio rapidly approaches 1 at higher pressures and independent of pressure..$^{\mathbf{1 0 2 6 - 2 8}}$ So, $\gamma$ is taken to be approximately 1 in this research.

The isothermal variation of density with pressure is presented in Fig. 6. As expected, the isothermal density of PEEK film increases obviously with the elevated pressures. However, the slope of isothermal density also increases with the elevated temperature, which indicated that the enclosed PEEK film

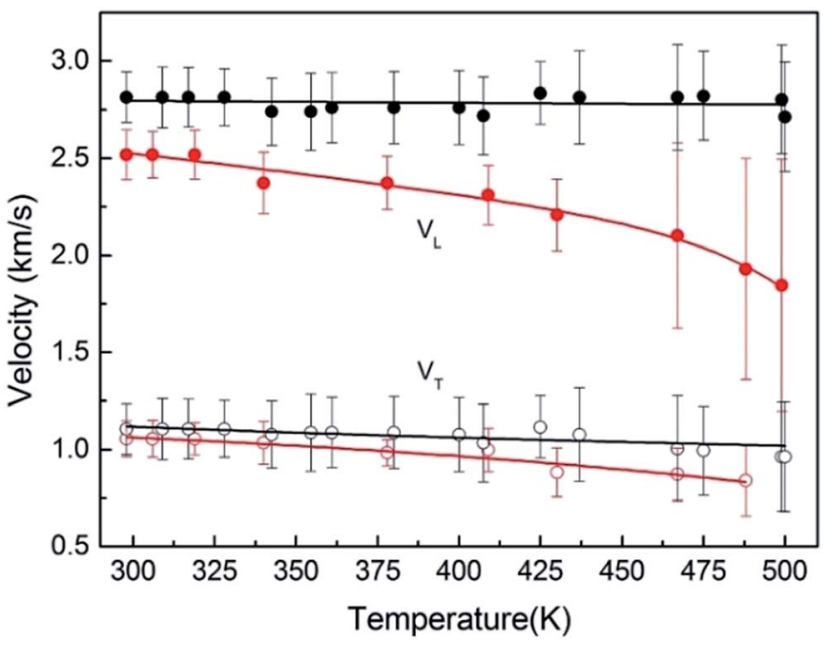

Fig. 4 The variation of sound velocity with temperature at $0 \mathrm{GPa}$ and $0.1 \mathrm{GPa}$, therein longitudinal velocity $\left(V_{\mathrm{L}}\right)$ and transverse velocity $\left(V_{\mathrm{T}}\right)$ under $0 \mathrm{GPa}$ are denoted by red roundness, the longitudinal velocity $\left(V_{\mathrm{L}}\right)$ and transverse velocity $\left(V_{\mathrm{T}}\right)$ below $0.1 \mathrm{GPa}$ are denoted by black roundness.

exhibits a higher density at a higher temperature under the constant high pressure.

Usually, there are different physical models to give universal relations between the pressure, volume, temperature and other parameters. Here, we tried fitting four different types of equation-of-state (EOS) forms, including third order BirchMurnaghan, Vinet, Tait and an empirical EOS form developed by Sun et al., these forms are given as follows:

$$
\begin{gathered}
p=\frac{3}{2} K_{0}\left[\left(\frac{V}{V_{0}}\right)^{-7 / 3}-\left(\frac{V}{V_{0}}\right)^{-5 / 3}\right] \\
\times\left[1+\left(\frac{3 K_{0}^{\prime}}{4}-3\right)\left(\left(\frac{V}{V_{0}}\right)^{-2 / 3}\right)-1\right] \\
p=3 K_{0}\left[\left(\frac{V}{V_{0}}\right)^{-2 / 3}-\left(\frac{V}{V_{0}}\right)^{-1 / 3}\right] \\
\times \exp \left[\frac{3}{2}\left(K_{0}^{\prime}-1\right)\left(1-\left(\frac{V}{V_{0}}\right)^{1 / 3}\right)\right] \\
p=B \exp \left[\frac{1}{C}\left(1-\frac{v}{v_{0}}\right)\right]-B \\
p=\frac{K_{0}}{(n-m)}\left[\left(\frac{v_{0}}{v}\right)^{n+1}-\left(\frac{v_{0}}{v}\right)^{m+1}\right] \quad(n=6.14, m=1.16)
\end{gathered}
$$

here, $v / v_{0}$ is the volumetric ratio at pressure $p$. For Tait EOS form (eqn (6)), the $B$ and $C$ are empirical parameters, and the ratio of these parameters $B / C$ is equal to the isothermal bulk modulus at ambient pressure $K_{0}$. For the Sun et al. EOS form, the parameters $n=6.14$ and $m=1.16$ are used. Both Tait and Sun EOS forms allow for determination for $K_{0}$ only.

The results of the EOS fittings are listed in Table 1 . All the EOS fittings give similar curves going through the experimental data except that of Sun EOS, which displays a little bit shallower 

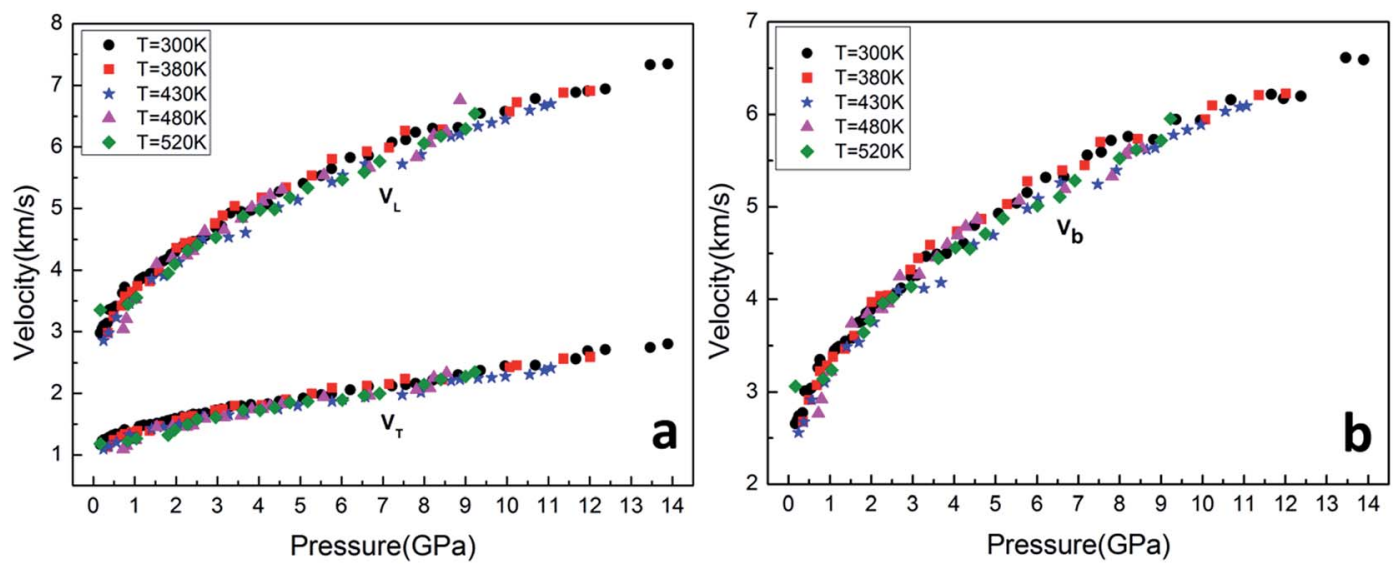

Fig. 5 Pressure dependence of velocities of PEEK with pressure along five isotherms; longitudinal velocity $\left(V_{\mathrm{L}}\right)$ and transverse velocity $\left(V_{T}\right)$ are shown in (a); the calculated bulk velocities are denoted in (b).

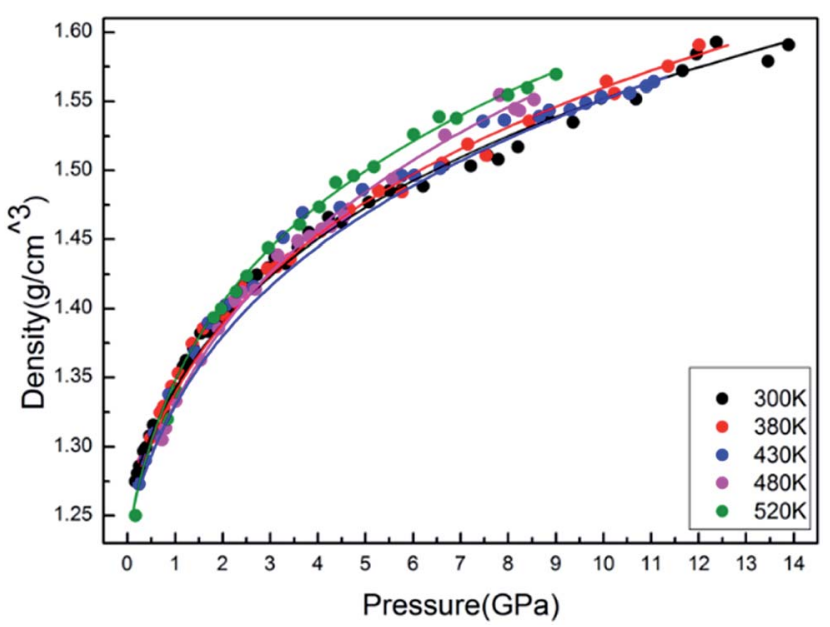

Fig. 6 Density variation with pressure for sample in five isotherms for PEEK film at 300, 380, 430, 480 and $520 \mathrm{~K}$. The solid ball are derived from the Brillouin measurements, Tait EOS forms fittings for the Brillouin data are presented at various temperatures.

fit to the isotherms in five isotherms. It is found that the Tait EOS fitting gives best results and is illustrated in the pressuredensity plot in Fig. 6. After analyzing of the isotherm determined from Brillouin scattering, we found that the $K_{0}$ and $K_{0}^{\prime}$ decrease with the increasing of temperature. The Sun EOS fitting shows a much higher $K_{0}$, while the third order BirchMurnaghan EOS fitting shows a lower $K_{0}$ and a much higher $K_{0}^{\prime}$.
From the EOS of PEEK film, we found that the PEEK film exhibits a higher density at higher temperature under a constant pressure, which is a typical negative thermal expansion phenomenon. This abnormal phenomenon would be explained by virtue of free volume theory. Usually, the PEEK film possesses plenty of free volume because of its conventional forming conditions ( $t \sim T_{\mathrm{g}}$; around ambient pressure). Under normal conditions, high temperature will supply more energy to the polymer chains for movement and achievement of more physical conformations, resulting a decreasing density. However, under the ultrahigh pressures, moveable polymer chains, soften by the high temperature, tend to gather and consume partial free volume. As a result, higher temperature brings about higher density of PEEK films under high pressures. This process seemed like the annealing technology in the polymer engineering, usually resulting in the drastic crystallization change. The study of crystallization change for PEEK film under extreme condition is currently undergoing in our lab, and will be presented in the future report.

With the densities calculated above, an analysis of elastic properties and individual elastic constants as a function of applied pressure were accomplished. The elasticity of isotropic materials is fully described by the elastic constants. The elastic moduli for all pressure and temperature, including $C_{11}, C_{12}$ and $C_{44}$, were calculated by the following equations.

$$
\rho V_{\mathrm{L}}^{2}=C_{11}
$$

Table 1 Isothermal bulk modulus $K_{0}$ and its pressure derivative $K^{\prime}{ }_{0}$ determined for sample using various EOS forms for five isotherms

\begin{tabular}{|c|c|c|c|c|c|c|c|c|c|c|}
\hline \multirow[b]{2}{*}{$T$} & \multicolumn{2}{|l|}{$300 \mathrm{~K}$} & \multicolumn{2}{|l|}{$380 \mathrm{~K}$} & \multicolumn{2}{|c|}{$430 \mathrm{~K}$} & \multicolumn{2}{|l|}{$480 \mathrm{~K}$} & \multicolumn{2}{|l|}{$520 \mathrm{~K}$} \\
\hline & $K_{0}$ & $K_{0}^{\prime}$ & $K_{0}$ & $K_{0}^{\prime}$ & $K_{0}$ & $K_{0}^{\prime}{ }_{0}$ & $K_{0}$ & $K_{0}^{\prime}{ }_{0}$ & $K_{0}$ & $K_{0}^{\prime}$ \\
\hline $\mathrm{BM}$ & 6.83 & 36.40 & 6.81 & 30.76 & 6.73 & 30.10 & 5.54 & 29.18 & 5.09 & 27.49 \\
\hline Vinet & 9.14 & 16.47 & 8.27 & 15.87 & 8.13 & 15.76 & 7.70 & 14.12 & 7.00 & 13.68 \\
\hline Sun & 16.91 & - & 14.64 & - & 14.49 & - & 11.49 & - & 10.09 & - \\
\hline
\end{tabular}




$$
\rho V_{\mathrm{b}}^{2}=\frac{C_{11}-C_{12}}{2}=C_{44}
$$

As shown in Fig. 7a, the isothermal elastic constant increases dramatically under applied pressure. However, the elastic constants reveal little change with temperature variation under the same pressure. Contrast with $C_{44}, C_{11}$ and $C_{12}$ of elastic constants are more sensitive to the pressure. Furthermore, these elastic constants allow us to calculate adiabatic bulk modulus $\left(K_{\mathrm{s}}\right)$, shear modulus $(G)$, and Young's modulus $(E)$, and Poisson's ratios $(\sigma)$ as a function of pressure according to the following equations:

$$
\begin{gathered}
K_{\mathrm{s}}=C_{11}+C_{44}=\rho\left(V_{\mathrm{L}}^{2}-\frac{4}{3} V_{\mathrm{T}}^{2}\right) \\
G=\frac{C_{11}-C_{12}}{2}=C_{44} \\
E=\frac{C_{44}\left(3 C_{11}-4 C_{44}\right)}{C_{11}-C_{44}}=\frac{\left(C_{11}-C_{12}\right)\left(C_{11}+2 C_{12}\right)}{C_{11}+C_{12}} \\
\sigma=\frac{V_{\mathrm{L}}{ }^{2}-2 V_{\mathrm{T}}^{2}}{2 V_{\mathrm{L}}{ }^{2}-2 V_{\mathrm{T}}{ }^{2}}
\end{gathered}
$$

All the calculated modulus at high pressure high temperature are presented in Fig. 7b. The moduli were found to dramatically increase for PEEK film under applied pressure but little change with the elevated temperatures. The bulk modulus revealed more sensitive to the pressure than the shear and Young's modulus. The enclosed PEEK film showed good bulk modulus about $35 \mathrm{GPa}$ at the pressure of $5 \mathrm{GPa}$ and $55 \mathrm{GPa}$ at pressure of $10 \mathrm{GPa}$, which indicated that the PEEK film possessed good compressibility. Furthermore, the slope of $K_{\mathrm{s}}$ is approximately twice of the slope of $E$ and approximately sixfold of the slope of $G$. However, the other enclosed polymers (such as poly(chlorotrifluoroethylene-co-vinylidene fluoride), ${ }^{7}$ polyimde, ${ }^{11}$ cross-linked poly(dimethylsiloxane), ${ }^{27}$ cross-linked terpolymer poly(ethylene-vinyl acetate-vinyl alcohol), ${ }^{27}$ and segmented thermoplastic poly(ester urethane) copolymer ${ }^{27}$ ) revealed relatively high Young's moduli. Moreover, the pressure dependence of elastic constants and moduli are fitted by a linear function at $300 \mathrm{~K}, Y=Y_{0}+a \times P$, giving $Y_{0}=11.726$, 5.536, 1.941; $a=4.388,2.083,0.731$ for $K_{\mathrm{s}}, E$ and $G$, and giving $Y_{0}=14.314,10.417,1.941 ; a=5.363,3.896,0.731$ for $C_{11}, C_{12}$ and $C_{44}$, respectively.

The ratios of bulk modulus to shear modulus $(K / G)$ as function of pressure are calculated and shown in Fig. 8, which was used to estimate the brittle and ductile behavior of materials. ${ }^{29}$ Usually, a high $K / G$ ratio means good ductility of the material, whereas a low value corresponds to the brittle essence. The critical value is considered about $1.75 .^{30-32}$ As shown in Fig. 8a, the value of $K / G$ at five isotherms is much higher than 1.75 , which means that the PEEK film is of ductile essence. Furthermore, the value of $K / G$ increases remarkably with applied pressure increasing from 0 to $8 \mathrm{GPa}$, which indicates that pressure would improve the ductility of PEEK film. However, the values of $K / G$ begin to decrease when the applied pressure exceeds $8 \mathrm{GPa}$, which indicates that excess pressure would damage the ductility of PEEK film.

Poisson's ratios is the ratio of the relative contraction strain (or transverse strain) normal to the applied load, which is an important parameter for the mechanical characteristics of materials. As shown in the Fig. 8b, the Poisson's ratios of the PEEK film under high temperature high pressure are disclosed from 0.25 to 0.5 , which is the characteristic for the polymer materials. ${ }^{33-35}$ The larger Poisson ratio between 0.404 and 0.436 signifies the better ductile of the PEEK film. When the values of $K / G$ are much higher than 1 , as well as Poisson's ratios approach 0.5 , the materials are considered extremely incompressible. The Poisson's ratios for the PEEK film appear to increase with the increase of pressure and reach to the maximum 0.43 at about 8
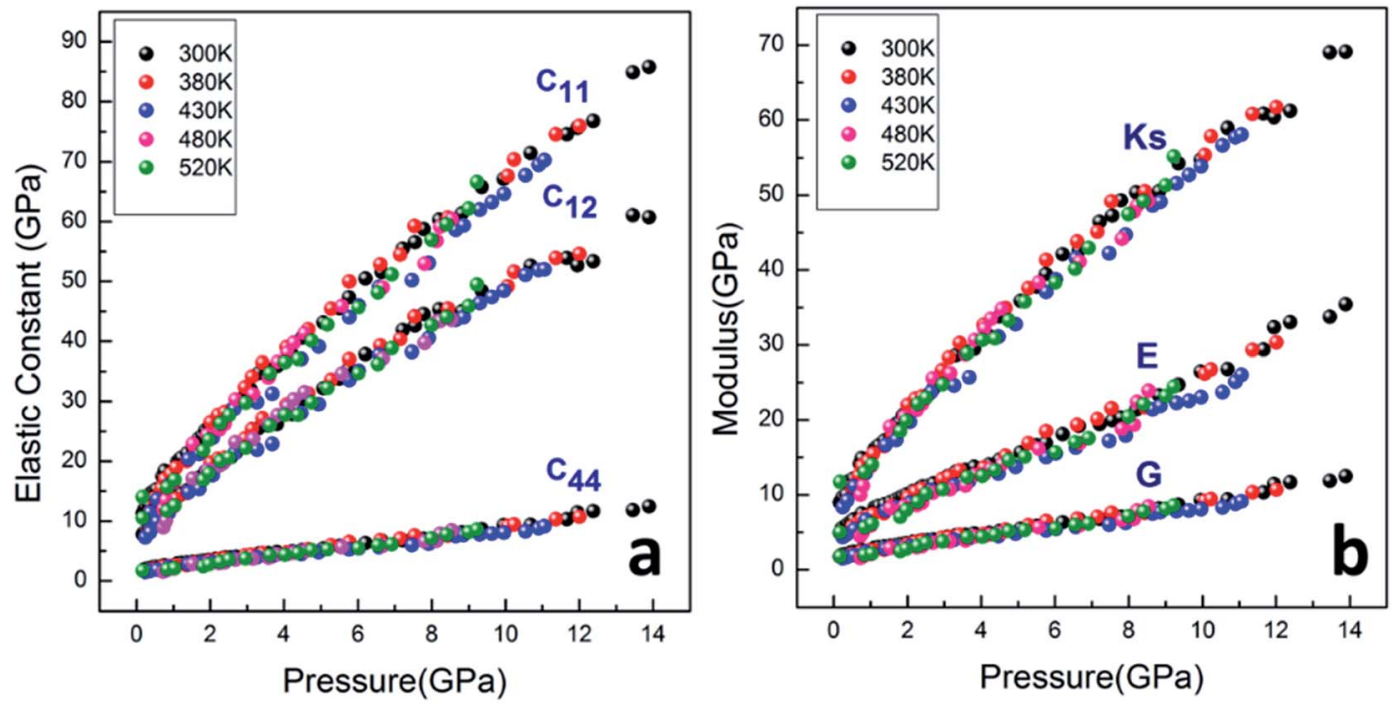

Fig. 7 (a) The five isotherms for PEEK film in the elastic constant-pressure plane include $C_{11}, C_{12}$ and $C_{44}$. (b) Elastic moduli as a function of pressure include bulk $(K)$, shear $(G)$, and Young's $(E)$ moduli vs. pressure in five isotherms. 

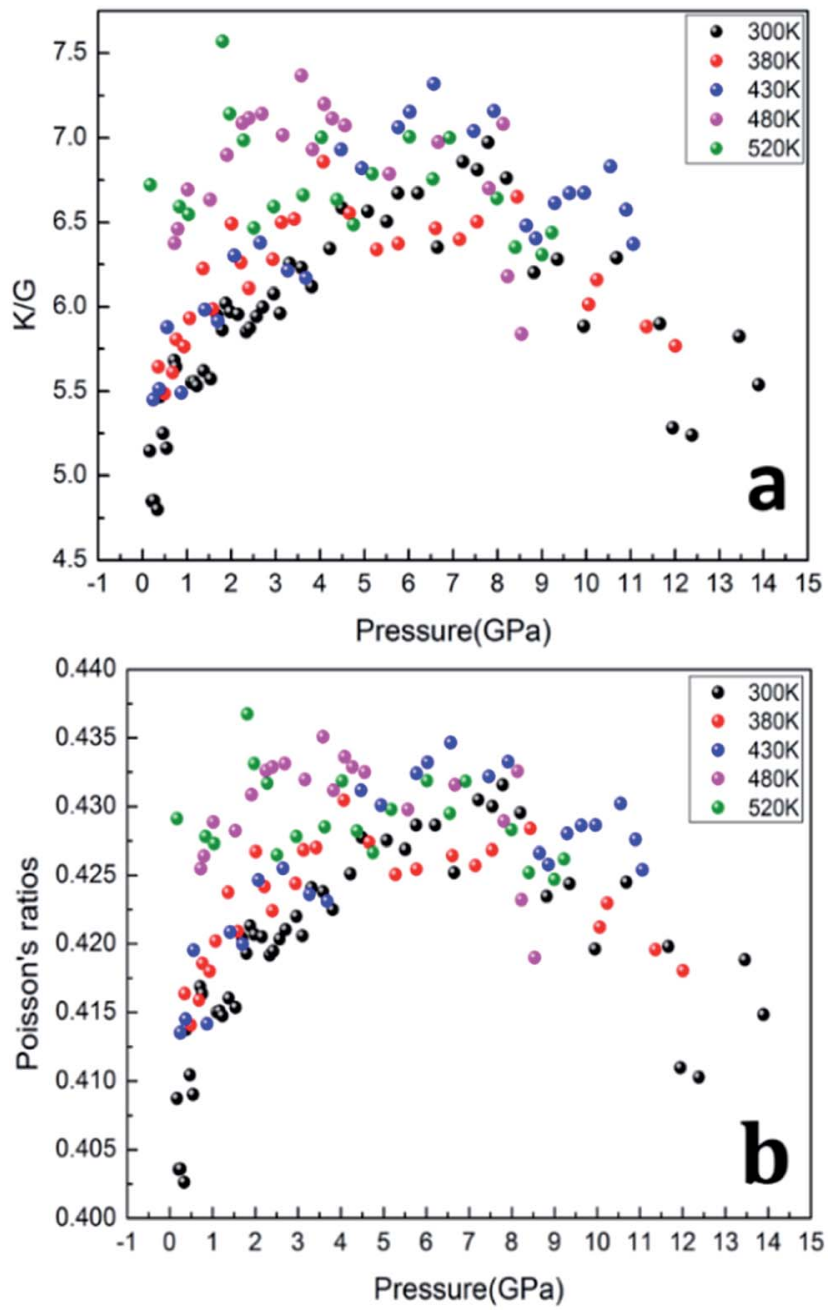

Fig. 8 Pressure dependence of $K / G(a)$ and Poisson's ratio (b) for PEEK film in five isotherms.

GPa. After that, the Poisson's ratios decrease obviously from 8 GPa to $13.9 \mathrm{GPa}$. Almost all of PEEK films on various isotherms reveal similar regularity. Moreover, the Poisson's ratios increase dramatically with the enhancing temperatures.

\section{Conclusions}

In conclusion, we have studied acoustic and elastic properties variation of PEEK film under high temperature high pressure by virtue of Brillouin scattering spectrum coupled with electrical resistance heating technique and diamond anvil cell device. The sound velocities, the elastic constants, bulk, shear, and Young's moduli, Poisson's ratios, density and their respective pressure dependencies were all disclosed and studied in detail. Free volume theory was also utilized to explain a negative thermal expansion phenomenon of PEEK film under high temperature high pressure. Our demonstrated system is expected to enrich the mechanical measurements of special engineering plastics and facilitates their practical applications under extreme conditions.

\section{Acknowledgements}

This work was supported by the National Natural Science Foundation of China (No. 11474127, 11574112), National Basic Research Program of China (No. 2011CB808200), Program for Changjiang Scholars and Innovative Research Team in University (No. IRT1132), National Found for Fostering Talents of basic Science (No. J1103202). We also acknowledge the support of Graduate Innovation Fund of Jilin University (2016147), and the Open Project of State Key Laboratory of Superhard Materials (Jilin University), China (Grant No. 201403).

\section{References}

1 K. Takamatsu, N. Ozaki, K. A. Tanaka, T. Onom, K. Nagai, M. Nakai, T. Watari, A. Sunahara, M. Nakano, T. Kataoka, H. Takenaka, M. Yoshida, K. Kondo and T. Yamanaka, Phys. Rev. E, 2003, 67, 056406-056410.

2 S. P. Marsh, LASL Shock Hugoniot Data, University of California Press, Berkeley, 1980.

3 S. Koda, T. Shibata, I. S. Park and S. Kojima, Curr. Appl. Phys., 2015, 15, 805-810.

4 L. Hong, B. Begen, A. Kisliuk, C. Alba-Simionesco, V. N. Novikov and A. P. Sokolov, Phys. Rev. B: Condens. Matter Mater. Phys., 2008, 78, 134201-134211.

5 S. Shin, J. H. Ko, J. Park, Y. H. Ko and K. J. Kim, Phys. B, 2015, 50, 466-467.

6 A. S. Benjamin, M. Ahart, S. A. Gramsch, L. L. Stevens, E. B. Orler, D. M. Dattelbaum and R. J. Hemley, J. Chem. Phys., 2012, 137, 014514-014520.

7 L. L. Stevens, D. M. Dattelbaum, M. Ahart and R. Hemeley, J. Appl. Phys., 2012, 112, 023523-023526.

8 H. Kriegs, G. Meier, J. Gapinski and A. Patkowski, J. Chem. Phys., 2008, 128, 014507-014515.

9 Z. A. Dreger, J. Zhou, N. C. Dang and Y. M. Gupta, J. Appl. Phys., 2011, 109, 083507-083576.

10 A. S. Benjamin, M. Ahart, S. A. Gramsch, L. L. Stevens, E. B. Orler, D. M. Dattelbaum and R. J. Hemley, J. Chem. Phys., 2012, 137, 014514-014517.

11 J. Wang, X. Zhao, E. B. Berda, C. Chen, K. Wang, S. Chen, B. Zou, B. Liu, Q. Zhou, F. Li and D. Chao, Polymer, 2016, 90, 1-8.

12 P. A. Rodgers, J. Appl. Polym. Sci., 1993, 48, 1061-1080.

13 G. R. Brannock and I. C. Sanchez, Macromolecules, 1993, 26, 4970-4972.

14 F. Yousefi, H. Karimi and M. Gomar, Fluid Phase Equilib., 2013, 355, 92-98.

15 R. B. Gupta and J. M. Prausnitz, Fluid Phase Equilib., 1996, 117, 77-83.

16 C. P. Bokis, M. D. Donohue and C. K. Hall, Ind. Eng. Chem. Res., 1994, 33, 1290-1298.

17 G. Bogdanic and A. Fredenslund, Ind. Eng. Chem. Res., 1994, 33, 1331-1340.

18 A. E. Wiącek, K. Terpiłowski, M. Jurak and M. Worzakowska, Eur. Polym. J., 2016, 78, 1-13.

19 S. M. Kurtz and J. N. P. Devine, Biomaterials, 2007, 28, 48454869. 
20 E. T. J. Rochford, A. H. C. Poulsson, J. Salavarrieta Varela, P. Lezuo, R. G. Richards and T. F. Moriarty, Colloids Surf., $B, 2014,113,213-222$.

21 D. D. Ragan, D. R. Clarke and D. Schiferl, Rev. Sci. Instrum., 1996, 67, 494-496.

22 Y. R. Shen, R. S. Kumar, M. Pravica and M. F. Nicol, Rev. Sci. Instrum., 2004, 75, 4450-4454.

23 X. Wang, C. Chen, X. Huang, J. Wang, M. Yao, K. Wang, F. Huang, B. Han, Q. Zhou and F. Li, RSC Adv., 2015, 5, 38056-38060.

24 F. Li, Q. Cui, Z. He, T. Cui, J. Zhang, Q. Zhou and G. Zou, J. Chem. Phys., 2005, 123, 174511-174514.

25 F. Li, M. Li, Q. Cui, T. Cui, Z. He, Q. Zhou and G. Zou, J. Chem. Phys., 2009, 131, 134502-134506.

26 J. F. Nye, Physical Properties of Crystals: Their Representation by Tensors and Matrices, Clarendon, Oxford, 1957, ch. 6 and 10 .
27 L. L. Stevens, E. B. Orler, D. M. Dattelbaum, M. Ahart and R. J. Hemley, J. Chem. Phys., 2007, 127, 104906-104918.

28 E. M. Brody, H. Shimizu, H. K. Mao, P. M. Bell and W. A. Bassett, J. Appl. Phys., 1981, 52, 3583-3585.

29 S. F. Pugh, Philos. Mag., 1954, 45, 823-843.

30 Y. Cao, J. Zhu, Z. Nong, X. Yang, Y. Liu and Z. Lai, Comput. Mater. Sci., 2013, 412, 208-213.

31 L. Qi, Y. Jin, Y. H. Zhao, X. Yang, H. Zhao and P. Han, J. Alloys Compd., 2015, 621, 383-388.

32 M. Pang, Y. Zhan, H. Wang, W. Jiang and Y. Du, J. Appl. Phys., 2011, 110, 033533-033539.

33 D. Chen, Z. Chen, M. L. Wang, N. H. Ma and H. W. Wang, Intermetallics, 2014, 52, 64-71.

34 G. N. Greaves, A. L. Greer, R. S. Lakes and T. Rouxel, Nat. Mater., 2011, 10, 823-837.

35 P. J. Rae and D. M. Dattelbaum, Polymer, 2004, 45, 76157625. 